\title{
Steady models of optically thin, magnetically supported two-temperature accretion disks around a black hole
}

\author{
H. Oda,${ }^{1}$ K. E. Nakamura, ${ }^{2}$ M. Machida ${ }^{3}$ and R. Matsumoto ${ }^{4}$ \\ ${ }^{1}$ Graduate School of Science and Technology, Chiba University, 1-33 Yayoi-Cho, Inage-ku, \\ Chiba 263-8522, Japan \\ ${ }^{2}$ Department of Sciences, Matsue National College of Technology, 14-4 Nishiikuma-Cho, \\ Matsue, Shimane 690-8518, Japan \\ ${ }^{3}$ Division of Theoretical Astronomy, National Astronomical Observatory of Japan, 2-21-1 \\ Osawa, Mitaka, Tokyo 181-8588, Japan \\ ${ }^{4}$ Department of Physics, Faculty of Science, Chiba University, 1-33 Yayoi-Cho, Inage-ku, \\ Chiba 263-8522, Japan \\ email: oda@astro.s.chiba-u.ac.jp, nakamrkn@matsue-ct.jp, mami@th.nao.ac.jp, \\ matumoto@astro.s.chiba-u.ac.jp
}

\begin{abstract}
We obtained steady solutions of optically thin two-temperature magnetized accretion disks around a black hole. We included relativistic bremsstrahlung cooling, synchrotron cooling and inverse Compton effects and assumed that the disk is threaded by toroidal magnetic field. We found that a magnetic pressure dominated new branch, which we call a 'low- $\beta$ branch', appears in the thermal equilibrium curves. The luminosity of the optically thin, magnetically supported disk can exceed $10 \%$ of the Eddington luminosity $\left(0.1 L_{\text {Edd }}\right)$.
\end{abstract}

Keywords. Accretion: accretion disks - black holes - state transitions - MHD

\section{Introduction}

X-ray hard state (low/hard state) of black hole candidates (BHCs) can be modeled by optically thin advection dominated accretion flows (ADAFs). Abramowicz et al. (1995) showed that when the accretion rate $\dot{M}$ exceeds a critical accretion rate $\dot{M}_{\text {c }}$, optically thin solution disappears. Above this critical accretion rate, the hard X-ray emitting optically thin hot accretion disk undergoes a transition to an optically thick cold disk which emit soft X-rays. RXTE observations of galactic BHCs revealed that some BHCs (e.g., XTE J1550-564) stay in X-ray hard state even when $L>0.1 L_{\text {Edd }}$ (e.g., Gierliński, Newton 2006). This luminosity is higher than the critical luminosity above which thermal instability takes place. Machida et al. (2006) carried out global 3D MHD simulations of this cooling instability and showed that magnetically supported disk is created.

\section{Models}

We extended the basic equations for 1D steady, axisymmetric, optically thin black hole accretion flows (e.g., Kato et al. 1998) by incorporating the azimuthal magnetic field. General relativistic effects are simulated by using pseudo-Newtonian potential (Paczyńsky \& Wiita 1980). We prescribe the vertically integrated $\varpi \varphi$-component of the stress tensor based on the results of local and global 3D MHD simulations (e.g., Machida et al. 2006; Hirose et al. 2006) as $T_{\varpi \varphi}=-\alpha_{\mathrm{B}} W$ and we set $\alpha_{\mathrm{B}}=0.05$. Machida et al. (2006) showed that $\int_{-\infty}^{\infty} v_{\varpi}\left\langle B_{\varphi}\right\rangle d z \propto \varpi^{-1}$ where $\left\langle B_{\varphi}\right\rangle$ is the azimuthally averaged 


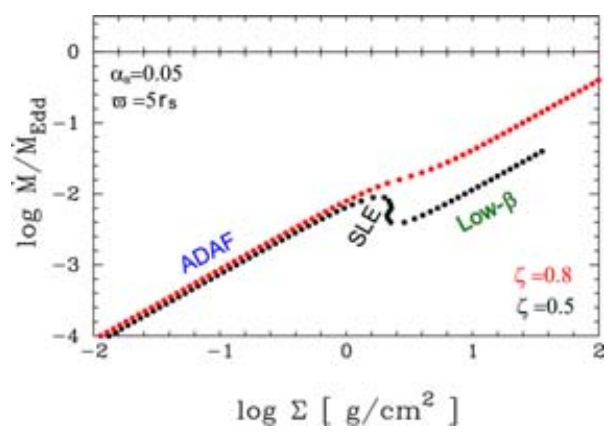

Figure 1. Local thermal equilibrium curves of optically thin disks at $\varpi=5 r_{\mathrm{s}}$ when $M=10 M_{\odot}$, $\alpha_{\mathrm{B}}=0.05$ and $\zeta=0.5$ (black) and $\zeta=0.8$ (red). We obtained a low- $\beta$ branch in addition to the ADAF and SLE branches. $\dot{M}_{\mathrm{Edd}}=4 \pi G M /\left(\eta_{\mathrm{e}} \kappa_{\mathrm{es}} c\right)$ is the Eddington accretion rate where $\eta_{\mathrm{e}}$ is the energy conversion efficiency taken to be $\eta_{\mathrm{e}}=0.1$.

toroidal field. Here we assume the radial advection rate of the toroidal magnetic field $\dot{\Phi}$ as

$$
\dot{\Phi}=-\int_{-\infty}^{\infty} v_{\varpi}\left\langle B_{\varphi}\right\rangle d z=-v_{\varpi} B_{0}(\varpi) \sqrt{4 \pi} H \equiv \dot{\Phi}_{\text {out }}\left(\frac{\varpi}{\varpi_{\text {out }}}\right)^{-\zeta}
$$

where $B_{0}(\varpi)=2^{5 / 4} \pi^{1 / 4}(\Re T / \mu)^{1 / 2} \Sigma^{1 / 2} H^{-1 / 2} \beta^{-1 / 2}$ is the strength of equatorial toroidal magnetic field and $\dot{\Phi}_{\text {out }}$ is the radial advection rate of the toroidal magnetic field at the outer boundary.

\section{Results}

We obtained local thermal equilibrium curves of optically thin, two-temperature black hole accretion flows at $\varpi=5 r_{\mathrm{s}}$ (figure 1 ). We found a low- $\beta$ branch in which the magnetic heating balances with the radiative cooling. The low- $\beta$ branch exists even when $\dot{M} \sim$ $0.5 \dot{M}_{\text {Edd }}$ and explains the luminous, X-ray hard state in BHCs observed during the transition from a low/hard state to a high/soft state. Since $T_{\mathrm{e}}<10^{9} \mathrm{~K}$ when $\dot{M}>\dot{M}_{\text {Edd }}$ in low- $\beta$ branch, the power-law X-ray spectrum in such disks becomes steeper and can explain the steep power law spectra observed in very high/steep power law state.

\section{Acknowledgements}

The authors thank Drs. M.A. Abramowicz, C. Akizuki, C. Done, J. Fukue, S. Hirose, Y. Kato, S. Mineshige, K. Ohsuga, R.A. Remillard, and K. Watarai for their valuable comments and discussions. This work was supported in part by the Grants-in-Aid for Scientific Research of the Ministry of Education, Culture, Sports, Science and Technology (RM: 17030003), Japan Society for the Promotion of Science (JSPS) Research Fellowships for Young Scientists (MM: 17-1907, 18-1907).

\section{References}

Abramowicz, M. A., Chen, X., Kato, S., Lasota, J.-P. \& Regev, O. 1995, ApJ, 438, L37

Gierliński, M. \& Newton, J. 2006, MNRAS, 370, 837

Hirose, S., Krolik, J. H. \& Stone, J. M. 2006, ApJ, 640, 901

Kato, S., Fukue, J. \& Mineshige, S. 1998, Black hole accretion disks (Kyoto: Kyoto University Press)

Machida, M., Nakamura, K. E. \& Matsumoto, R. 2006, PASJ, 58, 193

Paczyńsky, B. \& Wiita, P. J. 1980, A\&A, 88, 23 